Faculty of Mathematical Sciences

\section{University of Twente}

University for Technical and Social Sciences
P.O. Box 217

7500 AE Enschede

The Netherlands

Phone: +31-53-4893400

Fax: +31-53-4893114

Email: memo@math.utwente.nl

Memorandum No. 1567

The deviation matrix of a continuous-time

Markov chain

P. Coolen-Schrijner, ${ }^{1}$ And E.A. van Doorn

JANUARY 2001

ISSN 0169-2690

\footnotetext{
${ }^{1}$ Department of Mathematical Sciences, University of Durham, Science Laboratories, South Road, Durham DH1 3LE, United Kingdom
} 


\title{
The deviation matrix of a continuous-time
}

\author{
Markov chain
}

\author{
Pauline Coolen-Schrijner* and Erik A. van Doorn ${ }^{\dagger}$
}

*Department of Mathematical Sciences

University of Durham

Science Laboratories, South Road, Durham DH1 3LE, UK

$\dagger$ Faculty of Mathematical Sciences

University of Twente

P.O. Box 217, 7500 AE Enschede, The Netherlands

January 26, 2001

\begin{abstract}
The deviation matrix of an ergodic, continuous-time Markov chain with transition probability matrix $P($.$) and ergodic matrix \Pi$ is the matrix $D \equiv \int_{0}^{\infty}(P(t)-\Pi) d t$. We give conditions for $D$ to exist and discuss properties and a representation of $D$. The deviation matrix of a birth-death process is investigated in detail. We also describe a new application of deviation matrices by showing that a measure for the convergence to stationarity of a stochastically increasing Markov chain can be expressed in terms of the elements of the deviation matrix of the chain.
\end{abstract}

Keywords and phrases: birth-death process, convergence to stationarity, deviation matrix, ergodic Markov chain, ergodic potential, first entrance time, first return time, fundamental matrix, group inverse.

2000 Mathematics Subject Classification: Primary 60J27, Secondary 60J10 60J35 60J80 


\section{Introduction}

In what follows $\mathcal{X} \equiv\{X(t), t \geq 0\}$ is a time-homogeneous, continuous-time Markov chain with a discrete state space $S$ and governed by a standard stochastic matrix $P(t) \equiv\left(p_{i j}(t)\right)$ of transition probabilities

$$
p_{i j}(t) \equiv \operatorname{Pr}\{X(t)=j \mid X(0)=i\}, \quad i, j \in S, t \geq 0 .
$$

Throughout we will assume that $\mathcal{X}$ is ergodic, so that the limits

$$
\pi_{j} \equiv \lim _{t \rightarrow \infty} p_{i j}(t), \quad j \in S,
$$

exist, are strictly positive and independent of $i$, and constitute an honest distribution $\pi \equiv\left(\pi_{j}, j \in S\right)$, the ergodic distribution. This distribution can be obtained by solving the equation $\pi Q=0$, where $Q \equiv\left(q_{i j}\right)$ is the infinitesimal generator of $\mathcal{X}$. Evidently, $Q$ must be conservative, that is,

$$
-q_{i i}=q_{i} \equiv \sum_{j \neq i} q_{i j}, \quad i \in S
$$

The ergodic matrix $\Pi \equiv \lim _{t \rightarrow \infty} P(t)$ has all rows identical and equal to $\pi$, and satisfies

$$
\Pi^{2}=\Pi, \Pi Q=Q \Pi=0 \text { and } \Pi P(t)=P(t) \Pi=\Pi, t \geq 0 .
$$

The main object of interest in this paper is the matrix $D \equiv\left(d_{i j}\right)$ with elements

$$
d_{i j} \equiv \int_{0}^{\infty}\left(p_{i j}(t)-\pi_{j}\right) d t, \quad i, j \in S,
$$

which will be called the deviation matrix of $\mathcal{X}$, and which is conveniently represented as

$$
D=\int_{0}^{\infty}(P(t)-\Pi) d t
$$

$D$ is said to exist whenever all integrals in (1.2) exist and are finite. The deviation matrix is often referred to as fundamental matrix (see, for example, [10], [23] and [24]), but, parallelling well-established terminology for discretetime Markov chains, we will reserve this name for another (but related) matrix. Comments on terminology will be made in Section 3 . 
The purpose of this paper is to bring together and supplement a number of results on deviation matrices of continuous-time Markov chains which are scattered in the literature, in particular pertaining to the case in which $S$ is countably infinite. After briefly discussing the deviation matrix of a discretetime Markov chain and relating it to its continuous-time analogue in Section 2, we introduce more notation and terminology, and state some preliminary results in Section 3. Then, we will discuss the existence of $D$ in Section 4 and properties of $D$ in Section 5. The results in these sections constitute the continuous-time counterparts of results in Kemeny, Snell and Knapp [13, Chapter 9] on discretetime Markov chains, and supplement the findings of Syski in [23]. In Section 6 we will look in more detail into the deviation matrix of a birth-death process, thereby generalizing (and simplifying) the recent results of Koole and Spieksma [17]. Finally, in Section 7, we bring to light the role of the deviation matrix in a recently proposed criterion for the speed of convergence towards stationarity of the Markov chain $\mathcal{X}$.

\section{The discrete-time analogue}

In this section we introduce the discrete-time counterpart of (1.3) and show that it can be studied through the deviation matrix of a continuous-time Markov chain. So, let $\tilde{\mathcal{X}} \equiv\{\tilde{X}(n), n=0,1, \ldots\}$ be a discrete-time Markov chain on $S$ with matrix $\tilde{P}(n) \equiv\left(\tilde{p}_{i j}(n)\right)$ of $n$-step transition probabilities

$$
\tilde{p}_{i j}(n) \equiv \operatorname{Pr}\{\tilde{X}(n)=j \mid \tilde{X}(0)=i\}, \quad i, j \in S, n \geq 0,
$$

satisfying

$$
\tilde{P}(n)=\tilde{P}^{n}, \quad n \geq 0,
$$

where $\tilde{P}=\tilde{P}(1)$. We will assume that the chain is irreducible, positive recurrent and aperiodic. As a consequence the limits

$$
\tilde{\pi}_{j} \equiv \lim _{n \rightarrow \infty} \tilde{p}_{i j}(n), \quad j \in S
$$

exist, are strictly positive and independent of $i$, and constitute an honest distribution $\tilde{\pi} \equiv\left(\tilde{\pi}_{j}, j \in S\right)$ - the ergodic distribution of $\tilde{\mathcal{X}}$ - which satisfies the 
equation $\tilde{\pi}=\tilde{\pi} \tilde{P}$. The ergodic matrix $\tilde{\Pi} \equiv \lim _{n \rightarrow \infty} \tilde{P}(n)$ has all rows equal to $\tilde{\pi}$ and satisfies

$$
\tilde{\Pi} \tilde{P}=\tilde{P} \tilde{\Pi}=\tilde{\Pi}^{2}=\tilde{\Pi} .
$$

The deviation matrix of $\tilde{\mathcal{X}}$ is the matrix $\tilde{D} \equiv\left(\tilde{d}_{i j}\right)$ with elements

$$
\tilde{d}_{i j} \equiv \sum_{n=0}^{\infty}\left(\tilde{p}_{i j}(n)-\tilde{\pi}_{j}\right), \quad i, j \in S .
$$

$\tilde{D}$ can be represented as

$$
\tilde{D}=\sum_{n=0}^{\infty}(\tilde{P}(n)-\tilde{\Pi})
$$

and is said to exist whenever all sums in (2.4) exist and are finite.

We can associate with $\tilde{\mathcal{X}}$ a continuous-time Markov chain $\mathcal{X} \equiv\{X(t), t \geq 0\}$ with intensities

$$
q_{i j}=\tilde{p}_{i j}, \quad i \neq j, i, j \in S
$$

Since $\sum_{j \neq i} q_{i j}=\sum_{j \neq i} \tilde{p}_{i j}=1-\tilde{p}_{i i} \leq 1$ for all $i$, the process $\mathcal{X}$ is uniformizable with uniformization parameter 1 and we get $\tilde{\mathcal{X}}$ back as the uniformized process. Moreover, with $\{N(t), t \geq 0\}$ denoting a Poisson process with intensity 1, we have

$$
\{X(t), t \geq 0\} \stackrel{\mathrm{d}}{=}\{\tilde{X}(N(t)), t \geq 0\} .
$$

It follows in particular that both processes have the same ergodic distribution. (See, for example, Kijima [14, Section 4.4] for these results on uniformization.)

Writing

$$
\tilde{D}(\alpha)=\sum_{n=0}^{\infty}(\tilde{P}(n)-\tilde{\Pi}) \alpha^{n}, \quad 0<\alpha \leq 1,
$$

and

$$
D(\beta)=\int_{0}^{\infty} e^{-\beta t}(P(t)-\Pi) d t, \quad \beta \geq 0,
$$

and assuming that the deviation matrices $\tilde{D} \equiv \tilde{D}(1)$ of $\tilde{\mathcal{X}}$ and $D \equiv D(0)$ of $\mathcal{X}$ exist, it is clear from the Abelian Continuity Theorem that we must have

$$
\tilde{D}=\lim _{\alpha \uparrow 1} \tilde{D}(\alpha),
$$


and

$$
D=\lim _{\beta \downarrow 0} D(\beta) .
$$

Moreover, letting $\beta>0$ and $\alpha \equiv(1+\beta)^{-1}$, we can write

$$
\begin{aligned}
& D(\beta)=\int_{0}^{\infty} e^{-\beta t}(\tilde{P}(N(t))-\tilde{\Pi}) d t \\
& =\int_{0}^{\infty} e^{-\beta t} \sum_{n=0}^{\infty}(\tilde{P}(n)-\tilde{\Pi}) e^{-t} \frac{t^{n}}{n !} d t \\
& =\sum_{n=0}^{\infty}(\tilde{P}(n)-\tilde{\Pi}) \int_{0}^{\infty} e^{-(1+\beta) t} \frac{t^{n}}{n !} d t=\alpha \tilde{D}(\alpha),
\end{aligned}
$$

where we have employed (2.7) and Fubini's theorem. As a consequence we have $\tilde{D}=D$, as announced. The necessary and sufficient condition for the existence of the deviation matrix of a continuous-time Markov chain, obtained in Section 4 , and its discrete-time counterpart imply that $\tilde{D}$ and $D$ exist or do not exist together.

As an aside we remark that some authors use (2.10), or the Cesàro limit

$$
\tilde{D}=\lim _{N \rightarrow \infty} \frac{1}{N+1} \sum_{n=0}^{N}(\tilde{P}(n)-\tilde{\Pi})
$$

rather than (2.5) as a definition of the deviation matrix of a discrete-time Markov chain (see, for example, [3], [20], [17]). This has the advantage that one can allow periodicity of the Markov chain, provided the quantities $\tilde{\pi}_{j}$ of (2.2) are defined as Cesàro limits. We shall see in Section 4 that there is no advantage in defining the deviation matrix of a continuous-time Markov chain through (2.11) rather than (1.3).

\section{Preliminaries}

To motivate our terminology we recall some results from the theory of discretetime Markov chains (see Kemeny and Snell [11, Ch. 4]). So let $\tilde{\mathcal{X}}$ be the discrete-time Markov chain of the previous section and assume that its state space $S$ is finite. Then the inverse matrix

$$
\tilde{F}=(I-\tilde{P}+\tilde{\Pi})^{-1}
$$


exists and is called the fundamental matrix of $\tilde{\mathcal{X}}$. Since $\tilde{\mathcal{X}}$ is assumed to be aperiodic, $\tilde{F}$ has the representation

$$
\tilde{F}=\sum_{n=0}^{\infty}(\tilde{P}-\tilde{\Pi})^{n}
$$

which, in view of (2.1), (2.3) and (2.5), can be rewritten as

$$
\tilde{F}=\tilde{\Pi}+\sum_{n=0}^{\infty}(\tilde{P}(n)-\tilde{\Pi})=\tilde{\Pi}+\tilde{D} .
$$

The preceding was generalized to discrete-time Markov chains with discrete (finite or countably infinite) state space by Kemeny, Snell and Knapp [13, Ch. 9]. Indeed, if $S$ is countably infinite, the matrix (3.2) exists under certain conditions and satisfies (3.3) and

$$
\tilde{F}(I-\tilde{P}+\tilde{\Pi})=(I-\tilde{P}+\tilde{\Pi}) \tilde{F}=I .
$$

We now turn to the continuous-time Markov chain $\mathcal{X}$ of the Introduction. Without explicitly calling it a fundamental matrix the continuous-time analogue

$$
F=(\Pi-Q)^{-1}
$$

of (3.1) was introduced by Kemeny and Snell in [12] for finite $S$. In the same paper they state that

$$
F-\Pi=\int_{0}^{\infty}(P(t)-\Pi) d t(=D),
$$

and interpret this matrix "as a measure of the total deviation from the limiting probabilities". (Proofs of (3.6) can be found in Glynn [4] and Iosifescu [9]). Later Keilson [10], followed by Syski [22], [23] and others, used the name fundamental matrix to denote the matrix (3.6). Syski [22], [23] also refers to (3.6) as the ergodic potential (kernel). We prefer to maintain analogy in terminology between the discrete-time and continuous-time settings and thus, following the example of Iosifescu [9], reserve the name fundamental matrix for the matrix

$$
F \equiv D+\Pi
$$

with $D$ given by (1.3). Note that by defining $F$ in this way we can allow $S$ to be countably infinite. By using the name deviation matrix for the matrix $D$ we 
conform to the terminology propagated by Hordijk in a discrete-time setting (see, for example, [3], [7] and [20]).

When $S$ is finite the existence of $D$ (and $F$ ) is assured since for finite ergodic chains $p_{i j}(t)-\pi_{j}$ always has an exponential tail. Maintaining the assumption $S$ is finite, it is obvious from the definition of $D$ that

$$
D 1=0,
$$

and easy to see from the Kolmogorov differential equations that

$$
D Q=Q D=\Pi-I
$$

where $I$ denotes the identity matrix. With (1.1) it follows that

$$
(-Q) D(-Q)=-Q
$$

so that $D$ can be interpreted as a generalized inverse of $-Q$ (contrary to a statement in Yao [25]). Actually, since we also have

$$
D(-Q) D=D
$$

$D$ is the so-called group inverse (or Drazin inverse) of $-Q$, which, by definition, is the matrix solving the equations

$$
(-Q) X=X(-Q), X(-Q) X=X \text { and }(-Q) X(-Q)=-Q \text {. }
$$

Still assuming $S$ finite, the solution to this system, if it exists, is unique. For more information on generalized inverses and their application to Markov chains we refer to Meyer [19], Hunter [8] and Lamond and Puterman [18].

The existence of $D$ when $S$ is infinite will be our concern in Section 4 , and in Section 5 we investigate whether relations such as (3.8) and (3.9) remain valid in an infinite setting. A key role in the analysis will be played by the quantities $T_{i j}$, the first entrance time from state $i$ to state $j$ (so that $T_{j j}=0$ ). We let

$$
m_{i j}^{(k)} \equiv E\left(T_{i j}^{k}\right), \quad i, j \in S, k=1,2, \ldots,
$$

and $M^{(k)} \equiv\left(m_{i j}^{(k)}\right)$. By $T_{e j}$ we denote the first entrance time to $j$ from the ergodic distribution, and we let

$$
m_{e j}^{(k)} \equiv E\left(T_{e j}^{k}\right)=\sum_{i \in S} \pi_{i} m_{i j}^{(k)}, \quad j \in S, k=1,2, \ldots
$$


Finally, $R_{j}$ will denote the first return time to state $j$, and we let

$$
m_{j}^{(k)} \equiv E\left(R_{j}^{k}\right), \quad j \in S, k=1,2, \ldots
$$

We omit the index $(k)$ when $k=1$, that is, $m_{i j} \equiv m_{i j}^{(1)}, M \equiv M^{(1)}, m_{e j} \equiv m_{e j}^{(1)}$ and $m_{j} \equiv m_{j}^{(1)}$. In what follows we shall need the relation

$$
\sum_{\ell \in S} q_{i \ell} m_{\ell j}=-1+\delta_{i j} q_{j} m_{j}, \quad i, j \in S,
$$

which is given by Syski [23, eq. (1.12)], and follows from the Kolmogorov backward equations and (3.11) and (3.12) below. We shall also have use for the continuous-time counterparts of the Propositions 9.65, 9.67 and 9.68 in [13], which are collected in the next theorem.

Theorem 3.1 The following statements hold true for every $k=1,2, \ldots$ :

(i) $m_{j}^{(k)}<\infty$ for all $j \in S$ if and only if $m_{e j}^{(k-1)}<\infty$ for all $j \in S$;

(ii) $m_{j}^{(k)}<\infty$ for all $j \in S$ if and only if $m_{i j}^{(k)}<\infty$ for all $i, j \in S$;

(iii) if $m_{j}^{(k)}<\infty$ for some $j \in S$ then $m_{j}^{(k)}<\infty$ for all $j \in S$.

Proof. Writing $F_{i j}(t) \equiv \operatorname{Pr}\left\{T_{i j} \leq t\right\}$ and $F_{j}(t) \equiv \operatorname{Pr}\left\{R_{j} \leq t\right\}$, we have

$$
p_{i j}(t)=\int_{0}^{t} p_{j j}(t-s) d F_{i j}(s), \quad i \neq j, i, j \in S, t \geq 0,
$$

and

$$
p_{j j}(t)=e^{-q_{j} t}+\int_{0}^{t} p_{j j}(t-s) d F_{j}(s), \quad j \in S, t \geq 0,
$$

as is well known. Multiplying (3.11) with $\pi_{i}$ and summing over all $i \neq j$ subsequently gives us

$$
\pi_{j}\left(1-p_{j j}(t)\right)=\int_{0}^{t} p_{j j}(t-s) d F_{e j}(s), \quad j \in S, t \geq 0 .
$$

Now taking Laplace transforms in (3.12) and (3.13) we obtain, with obvious notation,

$$
\hat{p}_{j j}(s)=\frac{1}{s+q_{j}}+\hat{p}_{j j}(s) \hat{f}_{j}(s), \quad j \in S, s>0,
$$


and

$$
\frac{\pi_{j}}{s}-\pi_{j} \hat{p}_{j j}(s)=\hat{p}_{j j}(s) \hat{f}_{e j}(s), \quad j \in S, s>0 .
$$

Finally eliminating $\hat{p}_{j j}(s)$ from these equations yields

$$
s \hat{f}_{e j}(s)=\pi_{j} q_{j}-\pi_{j}\left(s+q_{j}\right) \hat{f}_{j}(s), \quad j \in S, s \geq 0,
$$

from which the result of statement $(i)$ readily follows.

The other two statements can be proven by arguments similar to those used in [13] in a discrete-time setting.

Evidently, $m_{j}=\left(q_{j} \pi_{j}\right)^{-1}<\infty$ for all $j \in S$ since $\mathcal{X}$ is ergodic. Hence, by the second statement of this theorem, $m_{i j}<\infty$ for all $i, j \in S$. On the other hand, $m_{e j}$ may be infinite when $S$ is infinite.

\section{Existence}

Syski [23, Proposition 3.2] states that the deviation matrix $D$ of $\mathcal{X}$ exists if

$$
m_{j}^{(2)}<\infty \text { and } m_{i j}<\infty \text { for all } i, j \in S \text {. }
$$

This result is weaker than related results for discrete-time Markov chains in Chapter 9 of [13] (see, in particular, Propositions 9.65, 9.68 and 9.75). However, it can be strengthened. Namely, Syski himself shows in the proof of his proposition that the condition (4.1) is actually sufficient and necessary for the (pointwise) existence (and finiteness) of

$$
\lim _{\beta \downarrow 0} D(\beta),
$$

where

$$
D(\beta) \equiv \int_{0}^{\infty} e^{-\beta t}(P(t)-\Pi) d t, \quad \beta \geq 0 .
$$

By applying a Tauberian theorem he subsequently shows that $D \equiv D(0)$ exists and coincides with (4.2). Since, on the other hand, the existence of $D$ implies the existence of the limit (4.2) (by the Abelian Continuity Theorem), it follows that condition (4.1) is necessary and sufficient for the existence of $D$. Now applying Theorem 3.1 we can conclude the following. 
Theorem 4.1 The deviation matrix $D$ of $\mathcal{X}$ exists if and only if $m_{e j}<\infty$ for some (and then for every) state $j \in S$.

We note that the arguments above show that there is no advantage in defining the deviation matrix of $\mathcal{X}$ by (4.2) rather than (1.3).

We conclude this section with the observation that the $M / G / 1$ example in Koole and Spieksma [17] provides a counter example to Syski's claim [23, p. 321] that boundedness of $Q$ is sufficient for the existence of $D$.

\section{Properties and representation}

We show in this section that properties such as (3.8) and (3.9) are valid also when $S$ is infinite, and give a formula expressing $D$ in terms of the first entrance and return time moments. Before doing so, however, we need a lemma which may be considered as the dual result of Proposition 3.6 in Syski [23]. The quantities $m_{i j}^{*}$ appearing in this lemma are the mean first entrance times of the reversed process $\mathcal{X}^{*} \equiv\left\{X^{*}(t), t \geq 0\right\}$, which is the process we obtain from $\mathcal{X}$ when we reverse time in a stationary version of $\mathcal{X}$. The reversed process is a Markov chain with rates

$$
q_{i j}^{*}=\frac{\pi_{j} q_{j i}}{\pi_{i}}, \quad i, j \in S,
$$

and transition probabilities $p_{i j}^{*}(t)$ satisfying

$$
p_{i j}^{*}(t)=\frac{\pi_{j} p_{j i}(t)}{\pi_{i}}, \quad i, j \in S,
$$

so that $\mathcal{X}^{*}$ is ergodic with ergodic distribution $\pi^{*}=\pi$. (See, for example, Anderson [1, p. 239]). Moreover, it is not difficult to see that (with obvious notation)

$$
m_{j}^{*}=m_{j} \text { and } m_{e j}^{*}=m_{e j}, \quad j \in S .
$$

(See [13, Proposition 9.70] for the discrete-time analogues.) It follows in particular that $D$ and the deviation matrix $D^{*}$ of $\mathcal{X}^{*}$ exist or do not exist together. The matrix $D(\beta)$ featuring in the lemma is defined in (4.3).

Lemma 5.1 If $D$ exists, and the vector $x \equiv\left(x_{i}, i \in S\right)$ satisfies the conditions 
(i) $\sum_{i \in S} \pi_{i}\left|x_{i}\right|<\infty$,

(ii) $\sum_{i \in S} \pi_{i}\left|x_{i}\right| m_{i j}^{*}<\infty, \quad j \in S$,

then $\lim _{\beta \downarrow 0}[D(\beta) x]=D x$.

Proof. Defining $\mu_{i} \equiv \pi_{i} x_{i}$ and $\mu \equiv\left(\mu_{i}, i \in S\right)$, and applying [23, Proposition 3.6] to $\mathcal{X}^{*}$, we obtain, with obvious notation

$$
\lim _{\beta \downarrow 0} \mu D^{*}(\beta)=\mu D^{*}
$$

Translating this result in terms of the original process $\mathcal{X}$ with the help of (5.2) yields the lemma.

We are now in a position to prove the next theorem, which is the continuoustime counterpart of the statements (2) - (4) of Proposition 9.76 in Kemeny, Snell and Knapp [13].

Theorem 5.2 When the deviation matrix $D$ of $\mathcal{X}$ exists, it has the properties

(i) $D 1=0$,

(ii) $D Q=Q D=\Pi-I$,

(iii) $D \Pi=\Pi D=0$.

Proof. If $D$ exists, then, by Theorem 4.1 and (5.3), the vector 1 satisfies the conditions of Lemma 5.1. Applying the lemma readily yields statement $(i)$.

The second equality in (ii) is given by Syski [23, eq. (4.5)] as an immediate consequence of his Theorem 4.2, but may also be viewed as a consequence of his equation (2.23) and the fact that

$$
\lim _{\beta \downarrow 0} Q D(\beta)=Q D,
$$

by his Proposition 3.6. To prove the first equality of $(i i)$ we choose $x_{i}=q_{i j}, i \in$ $S$, for some $j \in S$, and note that

$$
\sum_{i \in S} \pi_{i}\left|x_{i}\right| m_{i j}^{*}=\sum_{i \in S} \pi_{j} q_{j i}^{*} m_{i j}^{*}=\pi_{j}\left(-1+q_{j}^{*} m_{j}^{*}\right)<\infty,
$$


where we have used (5.1) and (3.10). Since $\pi Q=0$ it follows that every column of $Q$ satisfies the conditions of Lemma 5.1. Application of the lemma subsequently tells us that

$$
\lim _{\beta \downarrow 0}[D(\beta) Q]=D Q
$$

Finally, since $D(\beta) Q=Q D(\beta)$ for all $\beta>0$ by [23, eq. (2.23)], the first equality in $(i i)$ is implied by (5.4).

The second equality in statement (iii) is mentioned by Syski [23, eq. (3.15)] with the suggestion that a proof may be based on his Proposition 3.6. Indeed, as a consequence of this proposition we have

$$
\Pi D=\lim _{\beta \downarrow 0} \Pi D(\beta) .
$$

But (1.1) and Fubini's theorem tell us that $\Pi D(\beta)=0$ for all $\beta>0$. Evidently, the first equality in $(i i i)$ follows from the second and statement $(i)$.

Remarks. (i) Statement (i) is Corollary 3.3(b) in Syski [23], who suggests that it should follow directly from the definition of $D$. It is not clear to us, however, how the interchange of summation and integration can be justified without an auxiliary result such as Lemma 5.1.

(ii) Our proof of the first equality in statement (ii) shows that, contrary to a statement by Syski [23, Remark 2 on p. 327], no additional assumptions are required for the validity of $D Q=\Pi-I$ besides existence of $D$.

In $\left[23\right.$, Corollary 3.3] Syski shows that when the deviation matrix $D \equiv\left(d_{i j}\right)$ exists, it satisfies

$$
d_{i j}=d_{j j}-\pi_{j} m_{i j}, \quad i, j \in S
$$

with

$$
d_{j j}=\frac{1}{2} \frac{\pi_{j}}{m_{j}}\left(m_{j}^{(2)}-\pi_{j} m_{j}^{2}\right), \quad j \in S .
$$

Another representation for $d_{j j}$ can be obtained by observing that

$$
\sum_{i \in S} \pi_{i} d_{i j}=0, \quad j \in S
$$


by Theorem 5.1 (iii). It follows with (5.5) that

$$
d_{j j}=\pi_{j} \sum_{i \in S} \pi_{i} m_{i j}=\pi_{j} m_{e j}, \quad j \in S .
$$

Alternatively, we could have used (3.14) and (5.6) to obtain this result. (Note that $\hat{f}_{e j}(0)=1-\pi_{j}$.) Combining (5.5) and (5.7) we can write

$$
d_{i j}=\pi_{j}\left(m_{e j}-m_{i j}\right), \quad i, j \in S
$$

Thus we have obtained the next theorem, which is the continuous-time analogue of statement (5) of Proposition 9.76 in [13], and in which $\Pi_{d g}$ is the diagonal matrix with diagonal entries $\pi_{j}, j \in S$.

Theorem 5.3 When the deviation matrix $D \equiv\left(d_{i j}\right)$ of $\mathcal{X}$ exists, it satisfies

$$
D=(\Pi-I) M \Pi_{d g}
$$

\section{Birth-death processes}

The deviation matrix of a birth-death process has been studied by Whitt [24] (in a finite setting), and, recently, by Koole and Spieksma [17]. Actually, Koole and Spieksma consider the deviation matrix of a uniformized process, which requires the birth and death rates to be bounded. We will show that Whitt's results can straightforwardly be extended to birth-death processes with infinite state spaces (as remarked already by Whitt himself), thus generalizing and simplifying the results in [17].

In this section $\mathcal{X} \equiv\{X(t), t \geq 0\}$ will be an ergodic birth-death process taking values in $S=\{0,1, \ldots\}$ with birth rates $\left\{\lambda_{j}, j \in S\right\}$ and death rates $\left\{\mu_{j}, j \in S\right\}$, all strictly positive except $\mu_{0}=0$. We let

$$
\theta_{0} \equiv 1 \text { and } \theta_{j} \equiv \frac{\lambda_{0} \lambda_{1} \ldots \lambda_{j-1}}{\mu_{1} \mu_{2} \ldots \mu_{j}}, \quad j \geq 1 .
$$

Since $\mathcal{X}$ is ergodic these constants satisfy

$$
K \equiv \sum_{j=0}^{\infty} \theta_{j}<\infty,
$$

while

$$
\pi_{j} \equiv \lim _{t \rightarrow \infty} p_{i j}(t)=\frac{\theta_{j}}{K}, \quad j \in S .
$$


It is well known (see, for example, Kijima [14, p. 248]) that the mean first entrance times of $\mathcal{X}$ are given by

$$
m_{i j} \equiv E\left(T_{i j}\right)= \begin{cases}\sum_{k=i}^{j-1}\left(\lambda_{k} \pi_{k}\right)^{-1} \sum_{\ell=0}^{k} \pi_{\ell} & \text { if } i<j \\ \sum_{k=j}^{i-1}\left(\lambda_{k} \pi_{k}\right)^{-1} \sum_{\ell=k+1}^{\infty} \pi_{\ell} & \text { if } i \geq j,\end{cases}
$$

the empty sum being interpreted as zero. It follows in particular that

$$
m_{i 0}=\sum_{k=0}^{i-1}\left(\lambda_{k} \pi_{k}\right)^{-1}\left(1-\sum_{\ell=0}^{k} \pi_{\ell}\right), \quad i \in S,
$$

and hence, by interchanging summations,

$$
m_{e 0}=\sum_{i=0}^{\infty} \pi_{i} m_{i 0}=\sum_{k=0}^{\infty}\left(\lambda_{k} \pi_{k}\right)^{-1}\left(1-\sum_{\ell=0}^{k} \pi_{\ell}\right)^{2} .
$$

So, specifying Theorems 4.1 and 5.3 for the setting at hand, we obtain the following theorem, which extends Proposition 6 of Whitt [24] to a birth-death process with infinite state space.

Theorem 6.1 The deviation matrix $D \equiv\left(d_{i j}\right)$ of the birth-death process $\mathcal{X}$ exists if and only if $m_{e 0}<\infty$, that is,

$$
\sum_{k=0}^{\infty}\left(\lambda_{k} \pi_{k}\right)^{-1}\left(1-\sum_{\ell=0}^{k} \pi_{\ell}\right)^{2}<\infty
$$

in which case $d_{i j}$ satisfies (5.8) with $\pi_{j}$ and $m_{i j}$ given by (6.1)-(6.4).

For computational purposes it may be useful to observe that

$$
m_{i j}= \begin{cases}m_{0 j}-m_{0 i} & \text { if } i<j \\ m_{i 0}-m_{j 0} & \text { if } i \geq j\end{cases}
$$

because of the skip-free structure of a birth-death process, while

$$
m_{i 0}+m_{0 i}=\sum_{k=0}^{i-1}\left(\lambda_{k} \pi_{k}\right)^{-1}, \quad i \in S .
$$

It follows readily that

$$
m_{e j}=m_{e 0}-2 m_{j 0}+\sum_{k=0}^{j-1}\left(\lambda_{k} \pi_{k}\right)^{-1}, \quad j \in S,
$$


so that $d_{i j}$ can be expressed as

$$
d_{i j}=\pi_{j}\left(m_{e 0}-m_{i 0}-m_{j 0}+\sum_{k=0}^{\min \{i, j\}-1}\left(\lambda_{k} \pi_{k}\right)^{-1}\right), \quad i, j \in S,
$$

with $m_{e 0}$ given by (6.6) and the quantities $m_{i 0}$ by (6.5).

As an example we will apply the results of this section to the process of the number of customers in an $M / M / c$ queueing system, which is a birth-death process with rates

$$
\lambda_{j}=\lambda \text { and } \mu_{j}=\min \{j, c\} \mu, \quad j \in S .
$$

Writing

$$
\rho \equiv \frac{\lambda}{c \mu},
$$

we must have $\rho<1$ for the process to be ergodic. Substitution of (6.11) in (6.1)-(6.3) gives us

$$
\pi_{j}= \begin{cases}\frac{1}{K} \frac{(c \rho)^{j}}{j !} & 0 \leq j \leq c \\ \frac{1}{K} \frac{c^{c} \rho^{j}}{c !} & j \geq c,\end{cases}
$$

with

$$
K \equiv \sum_{j=0}^{c-1} \frac{(c \rho)^{j}}{j !}+\frac{1}{1-\rho} \frac{(c \rho)^{c}}{c !} .
$$

It is readily seen that

$$
m_{i 0}=\frac{1}{\lambda} \sum_{k=0}^{i-1} \frac{k !}{(c \rho)^{k}}\left\{\sum_{\ell=k+1}^{c-1} \frac{(c \rho)^{\ell}}{\ell !}+\frac{1}{1-\rho} \frac{(c \rho)^{c}}{c !}\right\}, \quad 0 \leq i \leq c,
$$

while

$$
m_{i 0}=m_{c 0}+(i-c) \frac{1}{\lambda} \frac{\rho}{1-\rho}, \quad i \geq c .
$$

As a consequence

$$
m_{e 0}=\frac{1}{K}\left\{\sum_{i=1}^{c-1} m_{i 0} \frac{(c \rho)^{i}}{i !}+\frac{1}{1-\rho} \frac{(c \rho)^{c}}{c !}\left(m_{c 0}+\frac{1}{\lambda} \frac{\rho^{2}}{(1-\rho)^{2}}\right)\right\},
$$

which is finite, so that the deviation matrix $D \equiv\left(d_{i j}\right)$ of the process exists for any $\rho<1$. It may subsequently be obtained by substituting $(6.13)-(6.17)$ in 
(6.10). The resulting expression is in accordance with [17, Theorem 4.2], but our derivation is simpler. Choosing $c=1$ gives us in particular

$$
d_{i j}=\frac{\rho^{\max \{0, j-i\}}-(i+j+1)(1-\rho) \rho^{j}}{\mu(1-\rho)}, \quad i, j \in S,
$$

a result which was obtained earlier by Syski [22] and Koole [16].

\section{An application}

Deviation matrices play an important role in perturbation theory for Markov chains ([22]), Markov decision processes ([3], [6], [17], [20]), simulation ([4], [24]), and first-passage time analysis and computation ([5], [25]). In this section we will describe a new application related to the speed of convergence to stationarity of a continuous-time Markov chain.

Throughout this section the ergodic Markov chain $\mathcal{X}$ takes values in $S=$ $\{0,1, \ldots\}$ and has $X(0)=0$. We will assume that $\mathcal{X}$ is stochastically increasing, so that $E(X(t))$ converges monotonically to its limiting value $E(X)$, with $X$ denoting a random variable with distribution $\left(\pi_{j}, j \in S\right)$. We will also assume

$$
E(X)=\sum_{j=0}^{\infty} j \pi_{j}<\infty,
$$

and shall be interested in the quantity

$$
m(\mathcal{X}) \equiv \int_{0}^{\infty}[1-E(X(t)) / E(X)] d t,
$$

which is positive, and may be interpreted as a measure of the speed of convergence to stationarity of $\mathcal{X}$.

Apart from normalization, (7.2) is the convergence measure proposed (and evaluated) by Stadje and Parthasarathy [21] in the setting of the $M / M / c$ queue. The present authors [2] have recently evaluated (7.2) for birth-death processes in general. In the (more general) setting at hand $m(\mathcal{X})$ can be expressed in terms of the elements of the deviation matrix of $\mathcal{X}$, as shown in the next theorem.

Theorem 7.1 If $m_{e 0}<\infty$ then

$$
m(\mathcal{X})=-\frac{1}{E(X)} \sum_{k=1}^{\infty} \sum_{j=k}^{\infty} d_{0 j}
$$

(which exists but may be infinite), whereas $m(\mathcal{X})=\infty$ otherwise. 
Proof. Since $\mathcal{X}$ is stochastically increasing, we have

$$
\sum_{j=0}^{k}\left(p_{0 j}(t)-\pi_{j}\right)>0, \quad k \geq 0 .
$$

We also observe

$$
E(X)-E(X(t))=\sum_{k=1}^{\infty} \sum_{j=k}^{\infty}\left(\pi_{j}-p_{0 j}(t)\right)=\sum_{k=1}^{\infty} \sum_{j=0}^{k-1}\left(p_{0 j}(t)-\pi_{j}\right) .
$$

It follows in particular that

$$
\int_{0}^{\infty}[E(X)-E(X(t))] d t \geq d_{00} \equiv \int_{0}^{\infty}\left(p_{00}(t)-\pi_{0}\right) d t .
$$

By (7.4), the integral on the right hand side of this inequality always exists, but may be infinite. Hence, by Theorem $4.1, m(\mathcal{X})=\infty$ if $m_{e 0}=\infty$. Now assuming $m_{e 0}<\infty$, and using (7.5) and Theorem $5.2(i)$, we can write

$$
\int_{0}^{\infty}[E(X)-E(X(t))] d t=\sum_{k=1}^{\infty} \sum_{j=0}^{k-1} d_{0 j}=-\sum_{k=1}^{\infty} \sum_{j=k}^{\infty} d_{0 j},
$$

the interchange of integration and summation being justified by (7.4) and Fubini's theorem. This proves the theorem.

With the help of a theorem on summation due to Markov (see Knopp [15, p. 250]) it can be shown that, actually,

$$
m(\mathcal{X})=-\frac{1}{E(X)} \sum_{j=0}^{\infty} j d_{0 j}<\infty
$$

if $\sum_{k=1}^{\infty} \sum_{j=k}^{\infty} d_{0 j}>-\infty$.

When $\mathcal{X}$ is the birth-death process of the previous section we observe from (6.10) that

$$
d_{0 j}=\pi_{j}\left(m_{e 0}-m_{j 0}\right), \quad j \in S,
$$

with $m_{j 0}$ and $m_{e 0}$ given by (6.5) and (6.6), respectively. It is now easy to obtain the following Corollary to Theorem 7.1, which is the result obtained earlier in $[2]$.

Corollary 7.2 If the Markov chain $\mathcal{X}$ is a birth-death process, then

$$
m(\mathcal{X})=\frac{1}{E(X)} \sum_{j=0}^{\infty} j \pi_{j} m_{j 0}-m_{e 0}
$$

if $\sum j \pi_{j} m_{j 0}<\infty$, whereas $m(\mathcal{X})=\infty$ otherwise. 


\section{References}

[1] W.J. Anderson (1991). Continuous-Time Markov Chains. Springer-Verlag, New York.

[2] P. Coolen-Schrijner and E.A. van Doorn (2000). On the convergence to stationarity of birth-death processes. Submitted.

[3] R. Dekker and A. Hordijk (1988). Average sensitive and Blackwell optimal policies in denumerable Markov decision chains with unbounded rewards. Math. Oper. Res. 13, 395-420.

[4] P. Glynn (1984). Some asymptotic formulas for Markov chains with applications to simulation. J. Statist. Comput. Simulation 19, 97-112.

[5] D.P. Heyman and D.P. O'Leary (1995). What is fundamental for Markov chains: First passage times, fundamental matrices, and group generalized inverses. In Computations with Markov Chains: Proceedings 2nd International Workshop on the Numerical Solution of Markov Chains, ed. W.J. Stewart. Kluwer, Boston, pp. 151-161.

[6] D.P. Heyman and D.P. O'Leary (1998). Overcoming instability in computing the fundamental matrix of a Markov chain. SIAM J. Matrix Anal. Appl. 19, 534-540.

[7] A. Hordijk and F.M. Spieksma (1994). A new formula for the deviation matrix. In Probability, Statistics and Optimisation - A Tribute to Peter Whittle, ed. F.P. Kelly. Wiley, Chichester, pp. 497-507.

[8] J.J. Hunter (1982). Generalized inverses and their application to applied probability problems. Linear Algebra Appl. 45, 157-198.

[9] M. Iosifescu (1980). Finite Markov Processes and Their Applications. Wiley, Chichester.

[10] J. Keilson (1971). Markov Chain Models: Rarity and Exponentiality. Springer-Verlag, New York. 
[11] J.G. Kemeny and J.L. Snell (1960). Finite Markov Chains. Van Nostrand, New York.

[12] J.G. Kemeny and J.L. Snell (1961). Finite continuous time Markov chains. Th. Probab. Appl. 6, 101-105.

[13] J.G. Kemeny, J.L. Snell and A.W. Knapp (1966). Denumerable Markov Chains. Van Nostrand, New York.

[14] M. Kijima (1997). Markov Processes for Stochastic Modeling. Chapman \& Hall, London.

[15] K. Knopp (1964). Theorie und Anwendung der Unendlichen Reihen, 5th ed. Springer, Berlin.

[16] G.M. Koole (1998). The deviation matrix of the $M / M / 1 / \infty$ and $M / M / 1 / N$ queue, with applications to controlled queueing models. Proceedings of the 37th IEEE CDC, Tampa, pp. 56-59.

[17] G.M. Koole and F.M. Spieksma (2001). On deviation matrices for birthdeath processes. Probab. Engng. Inform. Sci., to appear.

[18] B.F. Lamond and M.L. Puterman (1989). Generalized inverses in discrete time Markov decision processes. SIAM J. Matrix Anal. Appl. 10, 118-134.

[19] C.D. Meyer (1975). The role of the group generalized inverse in the theory of finite Markov chains. SIAM Rev. 17, 443-464.

[20] M.L. Puterman (1994). Markov Decision Processes. Wiley, New York.

[21] W. Stadje and P.R. Parthasarathy (1999). On the convergence to stationarity of the many-server Poisson queue. J. Appl. Probab. 36, 546-557.

[22] R. Syski (1977). Perturbation models. Stochastic Processes Appl. 5, 93-130.

[23] R. Syski (1978). Ergodic potential. Stochastic Processes Appl. 7, 311-336.

[24] W. Whitt (1992). Asymptotic formulas for Markov processes with applications to simulation. Operat. Res. 40, 279-291. 
[25] D.D. Yao (1985). First-passage-time moments of Markov processes. J. Appl. Probab. 22, 939-945. 